\title{
On split Regular Hom-Lie color algebras
}

\author{
Yan $\mathrm{Cao}^{1,2}$, Liangyun $\mathrm{Chen}^{1}$ \\ ${ }^{1}$ School of Mathematics and Statistics, Northeast Normal University, \\ Changchun 130024, China \\ 2 Department of Basic Education, Harbin University of Science and Technology, \\ Rongcheng Campus, Rongcheng 264300, China
}

\begin{abstract}
We introduce the class of split regular Hom-Lie color algebras as the natural generalization of split Lie color algebras. By developing techniques of connections of roots for this kind of algebras, we show that such a split regular Hom-Lie color algebra $L$ is of the form $L=U+\sum_{[j] \in \Lambda / \sim} I_{[j]}$ with $U$ a subspace of the abelian graded subalgebra $H$ and any $I_{[j]}$, a well described ideal of $L$, satisfying $\left[I_{[j]}, I_{[k]}\right]=0$ if $[j] \neq[k]$. Under certain conditions, in the case of $L$ being of maximal length, the simplicity of the algebra is characterized.
\end{abstract}

Key words: Hom-Lie color algebra, Lie color algebra, root system, root space MSC(2010): 17A32, 17A60, 17B22, 17B65

$\|$

\section{Introduction}

The notion of Hom-Lie algebras was introduced by Hartwig, Larsson and Silvestrov to describe the $q$-deformation of the Witt and the Virasoro algebras [1. Since then, many authors have studied Hom-type algebras [2 7]. The notion of Lie color algebras was introduced as generalized Lie algebras in 1960 by Ree [8]. So far, many results of this kind of algebras have been considered in the frameworks of enveloping algebras, representations and related problems [9-11]. In particular, Yuan introduced the notion of Hom-Lie color algebras in [12, which can be viewed as an extension of Hom-Lie (super)algebras to $\Gamma$-graded algebras, where $\Gamma$ is any abelian group.

Corresponding author(L. Chen): chenly640@nenu.edu.cn.

Supported by NNSF of China (Nos. 11171055 and 11471090), Scientific Research Fund of Heilongjiang Provincial Education Department (No. 12541184). 
As is well-known, the class of the split algebras is specially related to addition quantum numbers, graded contractions and deformations. For instance, for a physical system which displays a symmetry of $L$, it is interesting to know in detail the structure of the split decomposition because its roots can be seen as certain eigenvalues which are the additive quantum numbers characterizing the state of such system. Determining the structure of split algebras will become more and more meaningful in the area of research in mathematical physics. Recently, in [13 16], the structure of arbitrary split Lie algebras, arbitrary split Lie color algebras, arbitrary split Lie triple systems and arbitrary split regular HomLie algebras have been determined by the techniques of connections of roots. The purpose of this paper is to consider the structure of split regular Hom-Lie color algebras by the techniques of connections of roots based on some work in [14,15].

Throughout this paper, split regular Hom-Lie color algebras $L$ are considered of arbitrary dimension and over an arbitrary base field $\mathbb{K}$. This paper is organized as follows. In section 2, we establish the preliminaries on split regular Hom-Lie color algebras theory. In section 3, we show that such an arbitrary regular Hom-Lie color algebra $L$ with a symmetric root system is of the form $L=U+\sum_{[j] \in \Lambda / \sim} I_{[j]}$ with $U$ a subspace of the abelian subalgebra $H$ and any $I_{[j]}$ a well described ideal of $L$, satisfying $\left[I_{[j]}, I_{[k]}\right]=0$ if $[j] \neq[k]$. In section 4 , we show that under certain conditions, in the case of $L$ being of maximal length, the simplicity of the algebra is characterized.

\section{Preliminaries}

First we recall the definitions of Lie color algebras and Hom-Lie color algebras. The following definition is well-known from the theory of graded algebra.

Definition 2.1. 12] Let $\Gamma$ be an abelian group. A bi-character on $\Gamma$ is a map $\varepsilon: \Gamma \times \Gamma \rightarrow$ $\mathbb{K} \backslash\{0\}$ satisfying

1. $\varepsilon(\alpha, \beta) \varepsilon(\beta, \alpha)=1$,

2. $\varepsilon(\alpha, \beta+\gamma)=\varepsilon(\alpha, \beta) \varepsilon(\alpha, \gamma)$,

3. $\varepsilon(\alpha+\beta, \gamma)=\varepsilon(\alpha, \gamma) \varepsilon(\beta, \gamma)$,

for all $\alpha, \beta, \gamma \in \Gamma$.

It is clear that $\varepsilon(\alpha, 0)=\varepsilon(0, \alpha)=1$ for any $\alpha \in \Gamma$, where 0 denotes the identity element of $\Gamma$.

Definition 2.2. [15] Let $L=\oplus_{g \in \Gamma} L_{g}$ be a $\Gamma$-graded $\mathbb{K}$-vector space. For a nonzero homogeneous element $v \in L$, denote by $\bar{v}$ the unique group element in $\Gamma$ such that $v \in L_{\bar{v}}$, which will be called the homogeneous degree of $v$. We shall say that $L$ is a Lie color algebra if it is endowed with a $\mathbb{K}$-bilinear map $[\cdot, \cdot]: L \times L \rightarrow L$ satisfying

$$
\begin{aligned}
& {[v, w]=-\varepsilon(\bar{v}, \bar{w})[w, v],(\text { Skew- symmetry) }} \\
& {[v,[w, t]]=[[v, w], t]+\varepsilon(\bar{v}, \bar{w})[w,[v, t]], \text { (Jacobi identity) }}
\end{aligned}
$$


for all homogeneous elements $v, w, t \in L$.

Lie superalgebras are examples of Lie color algebras with $\Gamma=\mathbb{Z}_{2}$ and $\varepsilon(i, j)=(-1)^{i j}$, for any $i, j \in \mathbb{Z}_{2}$. We also note that $L_{0}$ is a Lie algebra.

Definition 2.3. [12] A Hom-Lie color algebra is a quadruple $(L,[\cdot, \cdot], \phi, \varepsilon)$ consisting of a $\Gamma$-graded space $L$, an even bilinear mapping $[\cdot, \cdot]: L \times L \rightarrow L$, a homomorphism $\phi$ and a bi-character $\varepsilon$ on $\Gamma$ satisfying

$$
\begin{aligned}
& {[x, y]=-\varepsilon(\bar{x}, \bar{y})[y, x],} \\
& \varepsilon(\bar{z}, \bar{x})[\phi(x),[y, z]]+\varepsilon(\bar{x}, \bar{y})[\phi(y),[z, x]]+\varepsilon(\bar{y}, \bar{z})[\phi(z),[x, y]]=0,
\end{aligned}
$$

for all homogeneous elements $x, y, z \in L, \bar{x}, \bar{y}, \bar{z}$ denote the homogeneous degree of $x, y, z$. When $\phi$ furthermore is an algebra automorphism it is said that $L$ is a regular Hom-Lie color algebra.

Clearly Hom-Lie algebras and Lie color algebras are examples of Hom-Lie color algebras.

Throughout this paper we will consider regular Hom-Lie color algebras $L$ being of arbitrary dimension and arbitrary base field $\mathbb{K}$. $\mathbb{N}$ denotes the set of all non-negative integers and $\mathbb{Z}$ denotes the set of all integers.

For any $x \in L$, we consider the adjoint mapping $\operatorname{ad}_{x}: L \rightarrow L$ defined by $\operatorname{ad}_{x}(z)=$ $[x, z]$. The usual regularity concepts will be understood in the graded sense. For instance, A subalgebra $A$ of $L$ is a graded subspace $I=\oplus_{g \in \Gamma} I_{g}$ of $L$ such that $[A, A] \subset A$ and $\phi(A)=A$. A graded subspace $I=\oplus_{g \in \Gamma} I_{g}$ of $L$ is called an ideal if $[I, L] \subset I$ and $\phi(I)=I$. A Hom-Lie color algebra $L$ will be called simple if $[L, L] \neq 0$ and its only (graded) ideals are 0 and $L$.

We introduce the concept of split regular Hom-Lie color algebra in an analogous way. We begin by considering a maximal abelian graded subalgebra $H=\oplus_{g \in \Gamma} H_{g}$ among the abelian graded subalgebras of $L$. Observe that $H$ is necessarily a maximal abelian subalgebra of $L$ as the following lemma shows.

Lemma 2.4. Let $H=\oplus_{g \in \Gamma} H_{g}$ be a maximal abelian graded subalgebra of a Hom-Lie color algebra $L$. Then $H$ is a maximal abelian subalgebra of $L$.

Proof. Consider an abelian subalgebra $K$ of $L$ such that $H \subset K$. For any $x \in K$ we have $\left[x, H_{g}\right]=0$ for each $g \in \Gamma$, and so by writing $x=\sum_{i=1}^{n} x_{g_{i}}$ with $x_{g_{i}} \in L_{g_{i}}$ for $i=1, \cdots, n$, being $g_{i} \in \Gamma$ and $g_{i} \neq g_{j}$ if $i \neq j$, we get by the grading $\left[x_{g_{i}}, H_{g}\right]=0$. Hence, for any $g_{i}, i=1, \cdots n$, we have $\left(H_{g_{i}}+\mathbb{K} x_{g_{i}}\right) \oplus\left(\oplus_{g \in \Gamma \backslash\left\{g_{i}\right\}} H_{g}\right)$ is an abelian graded subalgebra of $L$ containing $H$ and so $x_{g_{i}} \in H_{g_{i}}$. From here we get $x \in H$ and then $K=H$.

Let us introduce the class of split algebras in the framework of regular Hom-Lie color algebras. Denote by $H=\oplus_{g \in \Gamma} H_{g}$ a maximal abelian (graded) subalgebra, (MAGSA), of a regular Hom-Lie color algebra $L$. For a linear functional

$$
\alpha: H_{0} \rightarrow \mathbb{K}
$$


we define the root space of $L$ (with respect to $H$ ) associated to $\alpha$ as the subspace

$$
L_{\alpha}=\left\{v_{\alpha} \in L:\left[h_{0}, v_{\alpha}\right]=\alpha\left(h_{0}\right) \phi\left(v_{\alpha}\right) \text { for any } h_{0} \in H_{0}\right\} .
$$

The elements $\alpha: H_{0} \rightarrow \mathbb{K}$ satisfying $L_{\alpha} \neq 0$ are called roots of $L$ with respect to $H$. We denote $\Lambda:=\left\{\alpha \in H_{0}^{*} \backslash\{o\}: L_{\alpha} \neq 0\right\}$.

Definition 2.5. We say that $L$ is a split regular Hom-Lie color algebra, with respect to $H$, if

$$
L=H \oplus\left(\oplus_{\alpha \in \Lambda} L_{\alpha}\right) .
$$

We also say that $\Lambda$ is the root system of $L$.

Note that when $\phi=\mathrm{Id}$, the split Lie color algebras become examples of split regular Hom-Lie color algebras. Hence, the present paper extends the results in [15]. For convenience, the mappings $\left.\phi\right|_{H},\left.\phi\right|_{H} ^{-1}: H \rightarrow H$ will be denoted by $\phi$ and $\phi^{-1}$ respectively.

It is clear that the root space associated to the zero root $L_{o}$ satisfies $H \subset L_{o}$. Conversely, given any $v_{o} \in L_{o}$ we can write

$$
v_{o}=h \oplus\left(\oplus_{i=1}^{n} v_{\alpha_{i}}\right),
$$

where $h \in H$ and $v_{\alpha_{i}} \in L_{\alpha_{i}}$ for $i=1, \cdots, n$, with $\alpha_{i} \neq \alpha_{j}$ if $i \neq j$. Hence

$$
0=\left[h_{0}, h \oplus\left(\oplus_{i=1}^{n} v_{\alpha_{i}}\right)\right]=\oplus_{i=1}^{n} \alpha_{i}\left(h_{0}\right) \phi\left(v_{\alpha_{i}}\right),
$$

for any $h_{0} \in H_{0}$. So taking into account the direct character of the sum and that $\alpha_{i} \neq 0$ give us $v_{\alpha_{i}}=0$ for $i=1, \cdots, n$. So $v_{o}=h \in H$. Consequently,

$$
H=L_{o} .
$$

Lemma 2.6. Let $L=\oplus_{g \in \Gamma} L_{g}$ be a split Hom-Lie color algebra with corresponding root space decomposition $L=H \oplus\left(\oplus_{\alpha \in \Lambda} L_{\alpha}\right)$. If we denote by $L_{\alpha, g}=L_{\alpha} \cap L_{g}$, then the following assertions hold.

1. $L_{\alpha}=\oplus_{g \in \Gamma} L_{\alpha, g}$ for any $\alpha \in \Lambda \cup\{o\}$.

2. $H_{g}=L_{o, g}$. In particular $H_{0}=L_{o, 0}$.

3. $L_{0}$ is a split Hom-Lie algebra, respect to $H_{0}$, with root space decomposition $L_{0}=$ $H_{0} \oplus\left(\oplus_{\alpha \in \Lambda} L_{\alpha, 0}\right)$.

Proof. 1. By the $\Gamma$-grading of $L$ we may express any $v_{\alpha} \in L_{\alpha}, \alpha \in \Lambda \cup\{o\}$, in the form $v_{\alpha}=v_{\alpha, g_{1}}+\cdots+v_{\alpha, g_{n}}$ with $v_{\alpha, g_{i}} \in L_{g_{i}}$ for distinct $g_{1}, \cdots, g_{n} \in \Gamma$. If $h_{0} \in H_{0}$ then $\left[h_{0}, v_{\alpha, g_{i}}\right]=\alpha\left(h_{0}\right) \phi\left(v_{\alpha, g_{i}}\right)$ for $i=1, \cdots, n$. Hence $L_{\alpha}=\oplus_{g \in \Gamma}\left(L_{\alpha} \cap L_{g}\right)$ and we can write $L_{\alpha}=\oplus_{g \in \Gamma} L_{\alpha, g}$ for any $\alpha \in \Lambda \cup\{o\}$.

2. Consequence of Eq. (2.1) and item 1.

3. We also have $L_{g}=H_{g} \oplus\left(\oplus_{\alpha \in \Lambda} L_{\alpha, g}\right)$ for any $g \in \Gamma$. By considering $g=0$ we get $L_{0}=H_{0} \oplus\left(\oplus_{\alpha \in \Lambda} L_{\alpha, 0}\right)$. Hence, the direct character of the sum and the fact that $\alpha \neq 0$ for any $\alpha \in \Lambda$ give us that $H_{0}$ is a MASA of the Hom-Lie algebra $L_{0}$. Hence $L_{0}$ is a split Hom-Lie algebra respect to $H_{0}$. 
Lemma 2.7. For any $\alpha, \beta \in \Lambda \cup\{o\}$, the following assertions hold.

1. $\phi\left(L_{\alpha}\right) \subset L_{\alpha \phi^{-1}}$ and $\phi^{-1}\left(L_{\alpha}\right) \subset L_{\alpha \phi}$.

2. $\left[L_{\alpha}, L_{\beta}\right] \subset L_{\alpha \phi^{-1}+\beta \phi^{-1}}$.

Proof. 1. For $h_{0} \in H_{0}$ write $h_{0}^{\prime}=\phi\left(h_{0}\right)$. Then for all $h_{0} \in H_{0}$ and $v_{\alpha} \in L_{\alpha}$, since $\left[h_{0}, v_{\alpha}\right]=\alpha\left(h_{0}\right) \phi\left(v_{\alpha}\right)$, one has

$$
\left[h_{0}^{\prime}, \phi\left(v_{\alpha}\right)\right]=\phi\left(\left[h_{0}, v_{\alpha}\right]\right)=\alpha\left(h_{0}\right) \phi\left(\phi\left(v_{\alpha}\right)\right)=\alpha \phi^{-1}\left(h_{0}^{\prime}\right) \phi\left(\phi\left(v_{\alpha}\right)\right) .
$$

Therefore we get $\phi\left(v_{\alpha}\right) \in L_{\alpha \phi^{-1}}$ and so $\phi\left(L_{\alpha}\right) \subset L_{\alpha \phi^{-1}}$. In a similar way, one gets $\phi^{-1}\left(L_{\alpha}\right) \subset L_{\alpha \phi}$.

2. For any $h_{0} \in H_{0}, v_{\alpha} \in L_{\alpha}$ and $v_{\beta} \in L_{\beta}$, by denoting $h_{0}^{\prime}=\phi\left(h_{0}\right)$, by Hom-Jacobi identity, we have that

$$
\begin{aligned}
{\left[h_{0}^{\prime},\left[v_{\alpha}, v_{\beta}\right]\right] } & =\left[\left[h_{0}, v_{\alpha}\right], \phi\left(v_{\beta}\right)\right]+\varepsilon\left(\overline{h_{0}}, \overline{v_{\alpha}}\right)\left[\phi\left(v_{\alpha}\right),\left[h_{0}, v_{\beta}\right]\right] \\
& =\left[\alpha\left(h_{0}\right) \phi\left(v_{\alpha}\right), \phi\left(v_{\beta}\right)\right]+\beta\left(h_{0}\right)\left[\phi\left(v_{\alpha}\right), \phi\left(v_{\beta}\right)\right] \\
& =(\alpha+\beta)\left(h_{0}\right) \phi\left(\left[v_{\alpha}, v_{\beta}\right]\right) \\
& =(\alpha+\beta) \phi^{-1}\left(h_{0}^{\prime}\right) \phi\left(\left[v_{\alpha}, v_{\beta}\right]\right) .
\end{aligned}
$$

Therefore we get $\left[v_{\alpha}, v_{\beta}\right] \in L_{\alpha \phi^{-1}+\beta \phi^{-1}}$ and so $\left[L_{\alpha}, L_{\beta}\right] \subset L_{\alpha \phi^{-1}+\beta \phi^{-1}}$.

Lemma 2.8. If $\alpha \in \Lambda$ then $\alpha \phi^{-z} \in \Lambda$ for any $z \in \mathbb{Z}$.

Proof. It is a consequence of Lemma 2.7-1.

Definition 2.9. A root system $\Lambda$ of a split Hom-Lie color algebra is called symmetric if it satisfies that $\alpha \in \Lambda$ implies $-\alpha \in \Lambda$.

\section{Decompositions}

In the following, $L$ denotes a split regular Hom-Lie color algebra with a symmetric root system $\Lambda$ and $L=H \oplus\left(\oplus_{\alpha \in \Lambda} L_{\alpha}\right)$ the corresponding root decomposition. Given a linear functional $\alpha: H \rightarrow \mathbb{K}$, we denote by $-\alpha: H \rightarrow \mathbb{K}$ the element in $H^{*}$ defined by $(-\alpha)(h):=-\alpha(h)$ for all $h \in H$. We begin by developing the techniques of connections of roots in this section.

Definition 3.1. Let $\alpha$ and $\beta$ be two nonzero roots. We shall say that $\alpha$ is connected to $\beta$ if there exists $\alpha_{1}, \cdots, \alpha_{k} \in \Lambda$ such that

1. If $k=1$, then $\alpha_{1} \in\left\{a \phi^{-n}: n \in \mathbb{N}\right\} \cap\left\{ \pm \beta \phi^{-m}: m \in \mathbb{N}\right\}$. If $k \geq 2$, then $\alpha_{1} \in\left\{a \phi^{-n}: n \in \mathbb{N}\right\}$.

2. $\alpha_{1} \phi^{-1}+\alpha_{2} \phi^{-1} \in \Lambda$,

$$
\alpha_{1} \phi^{-2}+\alpha_{2} \phi^{-2}+\alpha_{3} \phi^{-1} \in \Lambda \text {, }
$$




$$
\begin{aligned}
& \alpha_{1} \phi^{-3}+\alpha_{2} \phi^{-3}+\alpha_{3} \phi^{-2}+\alpha_{4} \phi^{-1} \in \Lambda, \\
& \ldots \ldots \ldots \\
& \alpha_{1} \phi^{-i}+\alpha_{2} \phi^{-i}+\alpha_{3} \phi^{-i+1}+\cdots+\alpha_{i+1} \phi^{-1} \in \Lambda, \\
& \cdots \cdots \cdots \\
& \alpha_{1} \phi^{-k+2}+\alpha_{2} \phi^{-k+2}+\alpha_{3} \phi^{-k+3}+\cdots+\alpha_{i} \phi^{-k+i}+\cdots+\alpha_{k-1} \phi^{-1} \in \Lambda .
\end{aligned}
$$

3. $\alpha_{1} \phi^{-k+1}+\alpha_{2} \phi^{-k+1}+\alpha_{3} \phi^{-k+2}+\cdots+\alpha_{i} \phi^{-k+i-1}+\cdots+\alpha_{k} \phi^{-1} \in\left\{ \pm \beta \phi^{-m}: m \in \mathbb{N}\right\}$. We shall also say that $\left\{\alpha_{1}, \cdots, \alpha_{k}\right\}$ is a connection from $\alpha$ to $\beta$.

Observe that the case $k=1$ in Definition 3.1 is equivalent to the fact $\beta=\epsilon \alpha \phi^{z}$ for some $z \in \mathbb{Z}$ and $\epsilon \in\{ \pm 1\}$.

Lemma 3.2. For any $\alpha \in \Lambda$, we have that $\alpha \phi^{z_{1}}$ is connected to $\alpha \phi^{z_{2}}$ for every $z_{1}, z_{2} \in \mathbb{Z}$. We also have that $\alpha \phi^{z_{1}}$ is connected to $-\alpha \phi^{z_{2}}$ in case $-\alpha \phi^{z_{2}} \in \Lambda$.

Proof. This can be proved completely analogously to [14, Lemmas 2.2].

Lemma 3.3. Let $\left\{\alpha_{1}, \cdots, \alpha_{k}\right\}$ be a connection from $\alpha$ to $\beta$. Then the following assertions hold.

1. Suppose $\alpha_{1}=\alpha \phi^{-n}, n \in \mathbb{N}$. Then for any $r \in \mathbb{N}$ such that $r \geq n$, there exists $a$ connection $\left\{\bar{\alpha}_{1}, \cdots, \bar{\alpha}_{k}\right\}$ from $\alpha$ to $\beta$ such that $\bar{\alpha}_{1}=\alpha \phi^{-r}$.

2. Suppose that $\alpha_{1}=\epsilon \beta \phi^{-m}$ in case $k=1$ or

$$
\alpha_{1} \phi^{-k+1}+\alpha_{2} \phi^{-k+1}+\alpha_{3} \phi^{-k+2}+\cdots+\alpha_{k} \phi^{-1}=\epsilon \beta \phi^{-m}
$$

in case $k \geq 2$, with $m \in \mathbb{N}$ and $\epsilon \in\{ \pm 1\}$. Then for any $r \in \mathbb{N}$ such that $r \geq m$, there exists a connection $\left\{\bar{\alpha}_{1}, \cdots, \bar{\alpha}_{k}\right\}$ from $\alpha$ to $\beta$ such that $\bar{\alpha}_{1}=\epsilon \beta \phi^{-r}$ in case $k=1$ or

$$
\bar{\alpha}_{1} \phi^{-k+1}+\bar{\alpha}_{2} \phi^{-k+1}+\bar{\alpha}_{3} \phi^{-k+2}+\cdots+\bar{\alpha}_{k} \phi^{-1}=\epsilon \beta \phi^{-r}
$$

in case $k \geq 2$.

Proof. This can be proved completely analogously to [14, Lemmas 2.3].

Proposition 3.4. The relation $\sim$ in $\Lambda$, defined by $\alpha \sim \beta$ if and only if $\alpha$ is connected to $\beta$, is of equivalence.

Proof. This can be proved completely analogously to [14, Proposition 2.4].

For any $\alpha \in \Lambda$, we denote by

$$
\Lambda_{\alpha}:=\{\beta \in \Lambda: \beta \sim \alpha\}
$$

Clearly if $\beta \in \Lambda_{\alpha}$ then $-\beta \in \Lambda_{\alpha}$ and, by Proposition 3.4, if $\gamma \notin \Lambda_{\alpha}$ then $\Lambda_{\alpha} \cap \Lambda_{\gamma}=\emptyset$. 
Our next goal is to associate an adequate ideal $L_{\Lambda_{\alpha}}$ of $L$ to any $\Lambda_{\alpha}$. For $\Lambda_{\alpha}, \alpha \in \Lambda$, we define

$$
H_{\Lambda_{\alpha}}:=\operatorname{span}_{\mathbb{K}}\left\{\left[L_{\beta}, L_{-\beta}\right]: \beta \in \Lambda_{\alpha}\right\} .
$$

Then $H_{\Lambda_{\alpha}}$ is the direct sum of

$$
\sum_{\beta \in \Lambda_{\alpha}, g \in \Gamma}\left[L_{\beta, g}, L_{-\beta,-g}\right] \subseteq H_{0}
$$

and

$$
\sum_{\substack{\beta \in \Lambda_{\alpha} ; \\ g, g^{\prime} \in \Gamma, g+g^{\prime} \neq 0}}\left[L_{\beta, g}, L_{-\beta, g^{\prime}}\right] \subseteq \oplus_{g \in \Gamma \backslash\{0\}} H_{g} .
$$

We also define

$$
V_{\Lambda_{\alpha}}:=\oplus_{\beta \in \Lambda_{\alpha}} L_{\beta}=\oplus_{\beta \in \Lambda_{\alpha}, g \in \Gamma} L_{\beta, g} .
$$

Finally, we denote by $L_{\Lambda_{\alpha}}$ the following graded subspace of $L$,

$$
L_{\Lambda_{\alpha}}:=H_{\Lambda_{\alpha}} \oplus V_{\Lambda_{\alpha}}
$$

Proposition 3.5. For any $\alpha \in \Lambda$, the linear subspace $L_{\Lambda_{\alpha}}$ is a subalgebra of $L$.

Proof. First we have to check that $L_{\Lambda_{\alpha}}$ satisfies $\left[L_{\Lambda_{\alpha}}, L_{\Lambda_{\alpha}}\right] \subset L_{\Lambda_{\alpha}}$. Taking into account $H=L_{o}$, we have

$$
\left[L_{\Lambda_{\alpha}}, L_{\Lambda_{\alpha}}\right]=\left[H_{\Lambda_{\alpha}} \oplus V_{\Lambda_{\alpha}}, H_{\Lambda_{\alpha}} \oplus V_{\Lambda_{\alpha}}\right] \subset\left[H_{\Lambda_{\alpha}}, V_{\Lambda_{\alpha}}\right]+\left[V_{\Lambda_{\alpha}}, H_{\Lambda_{\alpha}}\right]+\Sigma_{\beta, \delta \in \Lambda_{\alpha}}\left[L_{\beta}, L_{\delta}\right] .
$$

Let us consider the first summand in (3.2). Note that $H_{\Lambda_{\alpha}}$ is the direct sum of

$$
\sum_{\beta \in \Lambda_{\alpha}, g \in \Gamma}\left[L_{\beta, g}, L_{-\beta,-g}\right] \subseteq H_{0} \subseteq L_{o}
$$

and

$$
\sum_{\substack{\beta \in \Lambda_{\alpha} ; \\ g, g^{\prime} \in \Gamma, g+g^{\prime} \neq 0}}\left[L_{\beta, g}, L_{-\beta, g^{\prime}}\right] \subseteq \oplus_{g \in \Gamma \backslash\{0\}} H_{g} \subseteq L_{o} .
$$

Given $\beta \in \Lambda_{\alpha}$, we have $\left[H_{\Lambda_{\alpha}}, L_{\beta}\right] \subset L_{\beta \phi^{-1}}$, being $\beta \phi^{-1} \in \Lambda_{\alpha}$ by Lemma 2.7-2. Hence,

$$
\left[H_{\Lambda_{\alpha}}, V_{\Lambda_{\alpha}}\right] \subset V_{\Lambda_{\alpha}}
$$

Similarly, we can also get

$$
\left[V_{\Lambda_{\alpha}}, H_{\Lambda_{\alpha}}\right] \subset V_{\Lambda_{\alpha}}
$$

Consider now the third summand $\Sigma_{\beta, \delta \in \Lambda_{\alpha}}\left[L_{\beta}, L_{\delta}\right]$. Given $\beta, \delta \in \Lambda_{\alpha}$ such that $\left[L_{\beta}, L_{\delta}\right] \neq 0$, if $\delta=-\beta$, then clearly $\left[L_{\beta}, L_{\delta}\right]=\left[L_{\beta}, L_{-\beta}\right] \subset H_{\Lambda_{\alpha}}$. Suppose that $\delta \neq-\beta$. Since $\left[L_{\beta}, L_{\delta}\right] \neq 0$ together with Lemma $2.7+2$ ensures that $\beta \phi^{-1}+\delta \phi^{-1} \in \Lambda$, we we have 
that $\{\beta, \delta\}$ is a connection from $\beta$ to $\beta \phi^{-1}+\delta \phi^{-1}$. The transitivity of $\sim$ gives now that $\beta \phi^{-1}+\delta \phi^{-1} \in \Lambda_{\alpha}$ and so

$$
\left[L_{\beta}, L_{\delta}\right] \subset L_{\beta \phi^{-1}+\delta \phi^{-1}} \subseteq V_{\Lambda_{\alpha}}
$$

From Eqs. (3.2)-(3.5) we conclude that $\left[L_{\Lambda_{\alpha}}, L_{\Lambda_{\alpha}}\right] \subset L_{\Lambda_{\alpha}}$.

Second, we have to verify that $\phi\left(L_{\Lambda_{\alpha}}\right)=L_{\Lambda_{\alpha}}$. But this is a direct consequence of Lemma 2.7-1 and Lemma 3.2 .

Proposition 3.6. If $\gamma \notin \Lambda_{\alpha}$ then $\left[L_{\Lambda_{\alpha}}, L_{\Lambda_{\gamma}}\right]=0$.

Proof. We have

$$
\left[L_{\Lambda_{\alpha}}, L_{\Lambda_{\gamma}}\right]=\left[H_{\Lambda_{\alpha}} \oplus V_{\Lambda_{\alpha}}, H_{\Lambda_{\gamma}} \oplus V_{\Lambda_{\gamma}}\right] \subset\left[H_{\Lambda_{\alpha}}, V_{\Lambda_{\gamma}}\right]+\left[V_{\Lambda_{\alpha}}, H_{\Lambda_{\gamma}}\right]+\left[V_{\Lambda_{\alpha}}, V_{\Lambda_{\gamma}}\right] .
$$

Consider the above third summand $\left[V_{\Lambda_{\alpha}}, V_{\Lambda_{\gamma}}\right]$ and suppose that there exist $\beta \in \Lambda_{\alpha}$ and $\eta \in \Lambda_{\gamma}$ such that $\left[L_{\beta}, L_{\eta}\right] \neq 0$. As necessarily $\beta \neq-\eta$, then $\beta \phi^{-1}+\eta \phi^{-1} \in \Lambda$. So $\left\{\beta, \eta,-\beta \phi^{-1}\right\}$ is a connection between $\beta$ and $\eta$. By the transitivity of the connection relation we have $\gamma \in \Lambda_{\alpha}$, a contradiction. Hence $\left[L_{\beta}, L_{\eta}\right]=0$ and so

$$
\left[V_{\Lambda_{\alpha}}, V_{\Lambda_{\gamma}}\right]=0
$$

Consider now the first summand $\left[H_{\Lambda_{\alpha}}, V_{\Lambda_{\gamma}}\right]$ in (3.6) and suppose there exist $\beta \in \Lambda_{\alpha}$ and $\eta \in \Lambda_{\gamma}$ such that $\left[\left[L_{\beta}, L_{-\beta}\right], \phi\left(L_{\eta}\right)\right] \neq 0$. Then

$$
\left[\left[L_{\beta, g}, L_{-\beta, g^{\prime}}\right], \phi\left(L_{\eta}\right)\right] \neq 0
$$

for some $g, g^{\prime} \in \Gamma$. By Hom Jacobi identity, either $\left[L_{-\beta, g^{\prime}}, \phi\left(L_{\eta}\right)\right] \neq 0$ or $\left[L_{\beta, g}, \phi\left(L_{\eta}\right)\right] \neq 0$ and so $\left[V_{\Lambda_{\alpha}}, V_{\Lambda_{\gamma}}\right] \neq 0$ in any case, what contradicts Eq. (3.7). Hence

$$
\left[H_{\Lambda_{\alpha}}, V_{\Lambda_{\gamma}}\right]=0
$$

Finally, we note that the same above argument shows

$$
\left[V_{\Lambda_{\gamma}}, H_{\Lambda_{\alpha}}\right]=0
$$

By Eq. (3.6) we conclude $\left[L_{\Lambda_{\alpha}}, L_{\Lambda_{\gamma}}\right]=0$.

Theorem 3.7. The following assertions hold.

1. For any $\alpha \in \Lambda$, the Hom-Lie color subalgebra

$$
L_{\Lambda_{\alpha}}=H_{\Lambda_{\alpha}} \oplus V_{\Lambda_{\alpha}}
$$

of $L$ associated to $\Lambda_{\alpha}$ is an ideal of $L$.

2. If $L$ is simple, then there exists a connection from $\alpha$ to $\beta$ for any $\alpha, \beta \in \Lambda$ and $H=\sum_{\alpha \in \Lambda}\left[L_{\alpha}, L_{-\alpha}\right]$. 
Proof. 1. Since $\left[L_{\Lambda_{\alpha}}, H\right]=\left[L_{\Lambda_{\alpha}}, L_{o}\right] \subset V_{[\alpha]}$, taking into account Propositions 3.5 and 3.6, we have

$$
\left[L_{\Lambda_{\alpha}}, L\right]=\left[L_{\Lambda_{\alpha}}, H \oplus\left(\oplus_{\beta \in \Lambda_{\alpha}} L_{\beta}\right) \oplus\left(\oplus_{\gamma \notin \Lambda_{\alpha}} L_{\gamma}\right)\right] \subset L_{\Lambda_{\alpha}}
$$

As we also have by Proposition 3.5 that $\phi\left(L_{\Lambda_{\alpha}}\right)=L_{\Lambda_{\alpha}}$, we conclude that $L_{\Lambda_{\alpha}}$ is an ideal of $L$.

2. The simplicity of $L$ implies $L_{\Lambda_{\alpha}}=L$. From here, it is clear that $\Lambda_{\alpha}=\Lambda$ and $H=\sum_{\alpha \in \Lambda}\left[L_{\alpha}, L_{-\alpha}\right]$.

Theorem 3.8. For a vector space complement $U$ of $\operatorname{span}_{\mathbb{K}}\left\{\left[L_{\alpha}, L_{-\alpha}\right]: \alpha \in \Lambda\right\}$ in $H$, we have

$$
L=U+\sum_{[\alpha] \in \Lambda / \sim} I_{[\alpha]},
$$

where any $I_{[\alpha]}$ is one of the ideals of $L$ described in Theorem 3.7 1, satisfying $\left[I_{[\alpha]}, I_{[\beta]}\right]=0$, whenever $[\alpha] \neq[\beta]$.

Proof. By Proposition 3.4, we can consider the quotient set $\Lambda / \sim:=\{[\alpha]: \alpha \in \Lambda\}$. let us denote by $I_{[\alpha]}:=L_{\Lambda_{\alpha}}$. We have $I_{[\alpha]}$ is well defined and, by Theorem $3.7-1$, an ideal of $L$. Therefore

$$
L=U+\sum_{[\alpha] \in \Lambda / \sim} I_{[\alpha]}
$$

By applying Proposition 3.6 we also obtain $\left[I_{[\alpha]}, I_{[\beta]}\right]=0$ if $[\alpha] \neq[\beta]$.

Definition 3.9. The annihilator of a Hom-Lie color algebra $L$ is the set $\mathrm{Z}(L)=\{x \in$ $L:[x, L]=0\}$.

Corollary 3.10. If $\mathrm{Z}(L)=0$ and $[L, L]=L$, then $L$ is the direct sum of the ideals given in Theorem 3.7 ,

$$
L=\oplus_{[\alpha] \in \Lambda / \sim} I_{[\alpha]} .
$$

Proof. From $[L, L]=L$, it is clear that $L=\oplus_{[\alpha] \in \Lambda / \sim} I_{[\alpha]}$. Finally, the sum is direct because $\mathrm{Z}(L)=0$ and $\left[I_{[\alpha]}, I_{[\beta]}\right]=0$ if $[\alpha] \neq[\beta]$.

\section{The simplicity of split regular Hom-Lie color alge- bras of maximal length.}

In this section we focus on the simplicity of split regular Hom-Lie color algebras by centering our attention in those of maximal length. From now on $\operatorname{char}(\mathbb{K})=0$.

Lemma 4.1. Let $L=H \oplus\left(\oplus_{\alpha \in \Lambda} L_{\alpha}\right)$ be a split regular Hom-Lie color algebra. If $I$ is an ideal of $L$ then $I=(I \cap H) \oplus\left(\oplus_{\alpha \in \Lambda}\left(I \cap L_{\alpha}\right)\right)$. 
Proof. We may view $L=H \oplus\left(\oplus_{\alpha \in \Lambda} L_{\alpha}\right)$ as a weight module respect to the split Hom-Lie color algebra $L_{0}$ with maximal abelian subalgebra $H_{0}$, (see Lemma 2.6-3), in the natural way. The characteristic property of ideals gives us that $I$ is a submodule of $L$. It is well-known that a submodule of a weight module is again a weight module. From here, $I$ is a weight module respect to $L_{0}$, (and $\left.H_{0}\right)$, and so $I=(I \cap H) \oplus\left(\oplus_{\alpha \in \Lambda}\left(I \cap L_{\alpha}\right)\right.$ ).

Taking into account the above lemma, observe that the grading of $I$ and Lemma 2.6-1 let us write

$$
I=\oplus_{g \in \Gamma} I_{g}=\oplus_{g \in \Gamma}\left(\left(I_{g} \cap H_{g}\right) \oplus\left(\oplus_{\alpha \in \Lambda}\left(I_{g} \cap L_{\alpha, g}\right)\right) .\right.
$$

Lemma 4.2. Let $L$ be a split regular Hom-Lie color algebra with $\mathrm{Z}(L)=0$ and $I$ an ideal of $L$. If $I \subset H$ then $I=\{0\}$.

Proof. Suppose there exists a nonzero ideal $I$ of $L$ such that $I \subset H$. We get $[I, H] \subset[H, H]=0$. We also get $\left[I, \oplus_{\alpha \in \Lambda} L_{\alpha}\right] \subset I \subset H$. Then taking into account $H=L_{o}$, we have $\left[I, \oplus_{\alpha \in \Lambda} L_{\alpha}\right] \subset H \cap\left(\oplus_{\alpha \in \Lambda} L_{\alpha}\right)=0$. From here $I \subset \mathrm{Z}(L)=0$, which is a contradiction.

Let us introduce the concepts of root-multiplicativity and maximal length in the framework of split Hom-Lie color algebras, in a similar way to the ones for split Hom Lie algebras (see [14]). For each $g \in \Gamma$, we denote by $\Lambda_{g}:=\left\{\alpha \in \Lambda: L_{\alpha, g} \neq 0\right\}$.

Definition 4.3. We say that a split regular Hom-Lie color algebra $L$ is root-multiplicative if given $\alpha \in \Lambda_{g_{i}}$ and $\beta \in \Lambda_{g_{j}}$, with $g_{i}, g_{j} \in \Gamma$, such that $\alpha+\beta \in \Lambda$, then $\left[L_{\alpha, g_{i}}, L_{\beta, g_{j}}\right] \neq 0$.

Definition 4.4. We say that a split regular Hom-Lie color algebra $L$ is of maximal length if for any $\alpha \in \Lambda_{g}, g \in \Gamma$, we have $\operatorname{dim} L_{\kappa \alpha, \kappa g}=1$ for $\kappa \in\{ \pm 1\}$.

Observe that if $L$ is of maximal lenth, then Eq. (4.8) let us assert that given any nonzero ideal $I$ of $L$ then

$$
I=\oplus_{g \in \Gamma}\left(\left(I_{g} \cap H_{g}\right) \oplus\left(\oplus_{\alpha \in \Lambda_{g}^{I}} L_{\alpha, g}\right)\right) .
$$

where $\Lambda_{g}^{I}:=\left\{\alpha \in \Lambda: I_{g} \cap L_{\alpha, g} \neq 0\right\}$ for each $g \in \Gamma$.

Theorem 4.5. Let $L$ be a split regular Hom-Lie color algebra of maximal length, root multiplicative and with $\mathrm{Z}(L)=0$. Then $L$ is simple if and only if it has all its nonzero roots connected and $H=\sum_{\alpha \in \Lambda}\left[L_{\alpha}, L_{-\alpha}\right]$.

Proof. The first implication is Theorem 3.7-2. To prove the converse,consider $I$ a nonzero ideal of $L$. By Lemma 4.2 and Eq. (4.9) we can write $I=\oplus_{g \in \Gamma}\left(\left(I_{g} \cap H_{g}\right) \oplus\right.$ $\left.\left(\oplus_{\alpha \in \Lambda_{g}^{I}} L_{\alpha, g}\right)\right)$ with $\Lambda_{g}^{I} \subset \Lambda_{g}$ for any $g \in \Gamma$ and some $\Lambda_{g}^{I} \neq \emptyset$. Hence, we may choose $\alpha_{0} \in \Lambda_{g}^{I}$ being so

$$
0 \neq L_{\alpha_{0}, g} \subset I .
$$

The fact $\phi(I)=I$ together with Lemma 2.7-1 allows us to assert that

$$
\text { if } \alpha \in \Lambda_{I} \text { then }\left\{\alpha \phi^{z}: z \in \mathbb{Z}\right\} \subset \Lambda_{I},
$$


that is

$$
\left\{L_{\alpha_{0} \phi^{z}, g}: z \in \mathbb{Z}\right\} \subset I .
$$

Now, let us take any $\beta \in \Lambda$ satisfying $\beta \notin\left\{ \pm \alpha_{0} \phi^{z}: z \in \mathbb{Z}\right\}$. Since $\alpha_{0}$ and $\beta$ are connected, we have a connection $\left\{\gamma_{1}, \gamma_{2}, \cdots, \gamma_{k}\right\}, k \geq 2$, from $\alpha_{0}$ to $\beta$ satisfying:

$$
\begin{aligned}
& \gamma_{1}=a_{0} \phi^{-n} \text { for some } n \in \mathbb{N} \text {, and } \\
& \gamma_{1} \phi^{-1}+\gamma_{2} \phi^{-1} \in \Lambda, \\
& \gamma_{1} \phi^{-2}+\gamma_{2} \phi^{-2}+\gamma_{3} \phi^{-1} \in \Lambda, \\
& \cdots \cdots \cdots \\
& \gamma_{1} \phi^{-i}+\gamma_{2} \phi^{-i}+\gamma_{3} \phi^{-i+1}+\cdots+\gamma_{i+1} \phi^{-1} \in \Lambda, \\
& \cdots \cdots \cdots \\
& \gamma_{1} \phi^{-k+2}+\gamma_{2} \phi^{-k+2}+\gamma_{3} \phi^{-k+3}+\cdots+\gamma_{i} \phi^{-k+i}+\cdots+\gamma_{k-1} \phi^{-1} \in \Lambda, \\
& \gamma_{1} \phi^{-k+1}+\gamma_{2} \phi^{-k+1}+\gamma_{3} \phi^{-k+2}+\cdots+\gamma_{i} \phi^{-k+i-1}+\cdots+\gamma_{k} \phi^{-1}=\epsilon \beta \phi^{-m} \text { for some } m \in \mathbb{N}
\end{aligned}
$$
and $\epsilon \in\{ \pm 1\}$.

Consider $\gamma_{1}, \gamma_{2}$ and $\gamma_{1}+\gamma_{2}$. Since $\gamma_{2} \in \Lambda$ there exists $g_{1} \in \Gamma$ such that $L_{\gamma_{2}, g_{1}} \neq 0$. From here, the root-multiplicativity and maximal length of $L$ show $0 \neq\left[L_{\gamma_{1}, g}, L_{\gamma_{2}, g_{1}}\right]=$ $L_{\left(\gamma_{1}+\gamma_{2}\right) \phi^{-1}, g+g_{1}}$, and by Eq. (4.12)

$$
0 \neq L_{\left(\gamma_{1}+\gamma_{2}\right) \phi^{-1}, g+g_{1}} \subset I .
$$

We can argue in a similar way from $\gamma_{1} \phi^{-1}+\gamma_{2} \phi^{-1}, \gamma_{3}$ and $\gamma_{1} \phi^{-2}+\gamma_{2} \phi^{-2}+\gamma_{3} \phi^{-1}$ to get

$$
0 \neq L_{\gamma_{1} \phi^{-2}+\gamma_{2} \phi^{-2}+\gamma_{3} \phi^{-1}, g_{2}} \subset I
$$

for some $g_{2} \in \Gamma$. Following this process with the connection $\left\{\gamma_{1}, \cdots, \gamma_{k}\right\}$ we obtain that

$$
0 \neq L_{\gamma_{1} \phi^{-k+1}+\gamma_{2} \phi^{-k+1}+\gamma_{3} \phi^{-k+2}+\cdots+\gamma_{k} \phi^{-1}, g_{3}} \subset I
$$

and so either $0 \neq L_{\beta \phi^{-m}, g_{3}} \subset I$ or $0 \neq L_{-\beta \phi^{-m}, g_{3}} \subset I$ for some $g_{3} \in \Gamma$. That is,

$$
0 \neq L_{\epsilon \beta \phi^{-m}, g_{3}} \subset I \quad \text { for } \quad \text { some } \quad \epsilon \in\{ \pm 1\}, \quad \text { some } \quad g_{3} \in \Gamma
$$

and for any $\beta \in \Lambda$. By Lemma 2.7-1, we can get

$$
0 \neq L_{\epsilon \beta, g_{3}} \subset I \quad \text { for } \quad \text { some } \quad \epsilon \in\{ \pm 1\}, \quad \text { some } \quad g_{3} \in \Gamma
$$

and for any $\beta \in \Lambda$.

Taking into account $H=\sum_{\gamma \in \Lambda}\left[L_{\gamma}, L_{-\gamma}\right]$, the grading of $L$ gives us

$$
H_{0}=\sum_{\gamma \in \Lambda, g \in \Gamma}\left[L_{\gamma, g}, L_{-\gamma,-g}\right] .
$$

From here, there exists $\gamma \in \Lambda$ and $g_{4} \in \Gamma$ such that

$$
\left[\left[L_{\gamma, g_{4}}, L_{-\gamma,-g_{4}}\right], \phi\left(L_{\epsilon \beta, g_{3}}\right)\right] \neq 0 .
$$


By the Hom Jacobi identity either $\left[L_{\gamma, g_{4}}, \phi\left(L_{\epsilon \beta, g_{3}}\right)\right] \neq 0$ or $\left[L_{-\gamma,-g_{4}}, \phi\left(L_{\epsilon \beta, g_{3}}\right)\right] \neq 0$ and so $L_{\gamma \phi^{-1}+\epsilon \beta \phi^{-2}, g_{4}+g_{3}} \neq 0$ or $L_{-\gamma \phi^{-1}+\epsilon \beta \phi^{-2},-g_{4}+g_{3}} \neq 0$. That is

$$
0 \neq L_{\kappa \gamma \phi^{-1}+\epsilon \beta \phi^{-2}, \kappa g_{4}+g_{3}} \subset I
$$

for some $\kappa \in\{ \pm 1\}$. Since $\epsilon \beta \in \Lambda_{g_{3}}$ we have by the maximal length of $L$ that $-\epsilon \beta \in \Lambda_{-g_{3}}$. By Eq. (4.16) and the root-multiplicativity and maximal length of $L$ we obtain

$$
0 \neq\left[L_{\kappa \gamma \phi^{-1}+\epsilon \beta \phi^{-2}, \kappa g_{4}+g_{3}}, L_{-\epsilon \beta \phi^{-2},-g_{3}}\right]=L_{\kappa \gamma \phi^{-2}, \kappa g_{4}} \subset I .
$$

By Lemma 2.7-1, we can get

$$
L_{\kappa \gamma, \kappa g_{4}} \subset I \text {. }
$$

Taking into account Eq. (4.17) and that Eq. (4.15) gives us

$$
\beta \phi^{-1}\left(\left[L_{\gamma, g_{4}}, L_{-\gamma,-g_{4}}\right]\right) \neq 0,
$$

we have that for any $g_{5} \in \Gamma$ such that $L_{\epsilon \beta, g_{5}} \neq 0$ necessarily

$$
0 \neq\left[\left[L_{\gamma, g_{4}}, L_{-\gamma,-g_{4}}\right], \phi\left(L_{\epsilon \beta, g_{5}}\right)\right]=L_{\epsilon \beta \phi^{-1}, g_{5}} \subset I
$$

and so $L_{\epsilon \beta \phi^{-1}} \subset I$. That is, we can assert that

$$
L_{\epsilon \beta} \subset I
$$

for any $\beta \in \Lambda$ and some $\epsilon \in\{ \pm 1\}$. Since $H=\sum_{\beta \in \Lambda}\left[L_{\beta}, L_{-\beta}\right]$ we get

$$
H \subset I \text {. }
$$

Now, given any $-\epsilon \beta \in \Lambda$, by the facts $-\epsilon \beta \neq 0, H \subset I$ and the maximal length of $L$ we have

$$
\left[H_{0}, L_{-\epsilon \beta}\right]=L_{-\epsilon \beta} \subset I \text {. }
$$

From Eqs. (4.19)-(4.21) we conclude that $I=L$. Consequently $L$ is simple.

Theorem 4.6. Let $L$ be a split regular Hom-Lie color algebra of maximal length, root multiplicative and satisfying $\mathrm{Z}(L)=0,[L, L]=L$. Then $L$ is the direct sum of the family of its minimal ideals, each one being a simple split regular Hom-Lie color algebra having all its nonzero roots connected.

Proof. By corollary [3.10, $L=\oplus_{[\alpha] \in \Lambda / \sim} I_{[\alpha]}$ is the direct sum of the ideals $I_{[\alpha]}=$ $H_{\Lambda_{\alpha}} \oplus V_{\Lambda_{\alpha}}=\left(\sum_{\beta \in[\alpha]}\left[L_{\beta}, L_{-\beta}\right]\right) \oplus\left(\oplus_{\beta \in[\alpha]} L_{\beta}\right)$ having any $I_{[\alpha]}$ its root system, $\Lambda_{\alpha}$, with all of its roots connected. It is easy to check that $\Lambda_{\alpha}$ has all of its roots $\Lambda_{\alpha}$-connected, (connected through roots in $\Lambda_{\alpha}$ ). We also have that any of the $I_{[\alpha]}$ is root-multiplicative as consequence of the root-multiplicativity of $L$. Clearly $I_{[\alpha]}$ is of maximal length, and finally $Z_{I_{[\alpha]}}\left(I_{[\alpha]}\right)=0$, (where $Z_{I_{[\alpha]}}\left(I_{[\alpha]}\right)$ ) denotes the center of $I_{[\alpha]}$ in $\left.I_{[\alpha]}\right)$, as consequence of $\left[I_{[\alpha]}, I_{[\beta]}\right] \stackrel{=}{=}$ if $[\alpha] \neq[\beta]$, (Theorem 3.8 ), and $Z(L)=0$. We can apply Theorem 4.5 to any $I_{[\alpha]}$ so as to conclude $I_{[\alpha]}$ is ssimple. It is clear that the decomposition $L=\bigoplus_{[\alpha] \in \Lambda / \sim} I_{[\alpha]}$ satisfies the assertions of the theorem. 


\section{References}

[1] J. Hartwig, D. Larsson and S. Silvestrov, (2006), Deformations of Lie algebras using $\sigma$-derivations. J. Algebra 295 (2), 314-361.

[2] F. Ammar, Z. Ejbehi and A. Makhlouf, (2011), Representations and cohomology of $n$-ary multiplicative Hom-Nambu-Lie algebras. J. Geom. Phys. 61 (10), 1898-1913.

[3] F. Ammar, S. Mabrouk and A. Makhlouf, (2011), Cohomology and deformations of Hom-algebras. J. Lie Theory 21 (4), 813-836.

[4] M. Elhamdadi, A. Makhlouf, (2011), Deformations of Hom-alternative and HomMalcev algebras. Algebras Groups Geom. 28 (2), 117-145.

[5] Q. Jin, X. Li, (2008), Hom-Lie algebra structures on semi-simple Lie algebras. J. Algebra 319(4), 1398-1408.

[6] A. Makhlouf, S. Silvestrov, (2010), Notes on 1-parameter formal deformations of Hom-associative and Hom-Lie algebras. Forum Math. 22 (4), 715-739.

[7] Y. Liu, L. Chen, Y. Ma, (2013), Hom-Nijienhuis operators and T*-extensions of hom-Lie superalgebras. Linear Algebra Appl. 439 (7), 2131-2144.

[8] R. Ree, (1960), Generalized Lie elements. Canad. J. Math. 12, 439-502.

[9] M. C. Wilson, (1995), Delta methods in enveloping algebras of Lie color algebras. J. Algebra. 75, 661-696.

[10] J. Bergen, D. S. Passman, (1995), Delta ideal of Lie color algebras. J. Algebra. 177, 740-754.

[11] J. Feldvoss, (2001), Representations of Lie color algebras. Adv. Math. 157, 95-137.

[12] L.Yuan, (2010), Hom-Lie color algebra structures, Comm. Algebra. 40 (2), 575-592.

[13] A. J. Calderón, (2008), On split Lie algebras with symmetric root systems. Proc. Indian Acad. Sci. (Math. Sci.) 118 (3), 351-356.

[14] M. J. Aragón, A. J. Calderón, (2015), Split regular Hom-Lie algebras. Journal of Lie Theory. 25 (3), 875-888.

[15] A. J. Calderón, J. M. Sánchez, (2012), On the structure of split Lie color algebras. Linear Algebra Appl. 436 (2), 307-315.

[16] A. J. Calderón, (2009), On split Lie triple systems. Proc. Indian Acad. Sci. (Math. Sci.) $119(2), 165-177$. 